\title{
Primary, exercise induced internal jugular vein thrombosis - A case report
}

\author{
Sergei Mashkov ${ }^{1 *}$, Gerard Bonnet ${ }^{2}$ and Medhat Osman ${ }^{3}$ \\ ${ }^{1}$ Surgical registrar, General Surgery, Whanganui DHB, New Zealand \\ ${ }^{2}$ General Surgeon, General Surgery Whanganui DHB, New Zealand \\ ${ }^{3}$ Radiologist, Whanganui District Health Board, New Zealand
}

\section{Introduction}

Internal jugular vein thrombosis (IJVT) is a rare but potentially fatal condition that can lead to pulmonary embolism and septicaemia [1].

IJVT is divided in 2 broad categories - primary and secondary [2]. Primary IJVTis associated with thoracic outlet syndrome (TOS), effort thrombosis and idiopathic thrombosis, when no obvious cause is identified [1]. Secondary IJVT is associated with trauma, surgery, malignancy, central venous catheterisation and coagulopathies.

Multiple studies reveal that primary IJVT tends to occur more often in women with a median age of 46 without significant illnesses or risk factors for cardiovascular disease [1].

Our patient presented with primary, exercise induced internal jugular and subclavian vein thrombosis which is very rare.

\section{Case report}

A 44 years old female with history hypothyroidism, on levothyroxin, presented to the emergency department with 2 days of pain, swelling and redness in the left lateral aspect of her neck and pain on abduction of her left shoulder. There was no history of recent trauma, malignant disease, oropharyngeal infection, operation or IV catheterisation. However, she admitted exercising for an hour on a stationary rowing machine a few days prior to her presentation.

On examination there was no regional lymphadenopathy, no axillary tenderness, and no thyroid enlargement. Examination of other systems were unremarkable. Neurogenic thoracic outlet syndrome tests, "the overhead stress test" and "the downward pull test" [3] were negative.

CT neck and chest followed by US of the left upper limb veins were negative for cervical rib and venous TOS, however revealed thrombosis in the distal left internal jugular vein, at the junction to the left subclavian and left brachiocephalic veins (Figure 1).

Blood results had shown moderately increased inflammatory markers. Coagulation studies including thrombophilia screen, anti cardiolipin and lupus anticoagulantserological were all negative.

A 5 days course of therapeutic Enoxaparin was administered and then she commenced Dabigatran for 3 months.

A CT scan 3 month from the initial presentation revealed complete resolution of thrombosis within the left internal jugular vein (Figure 2).

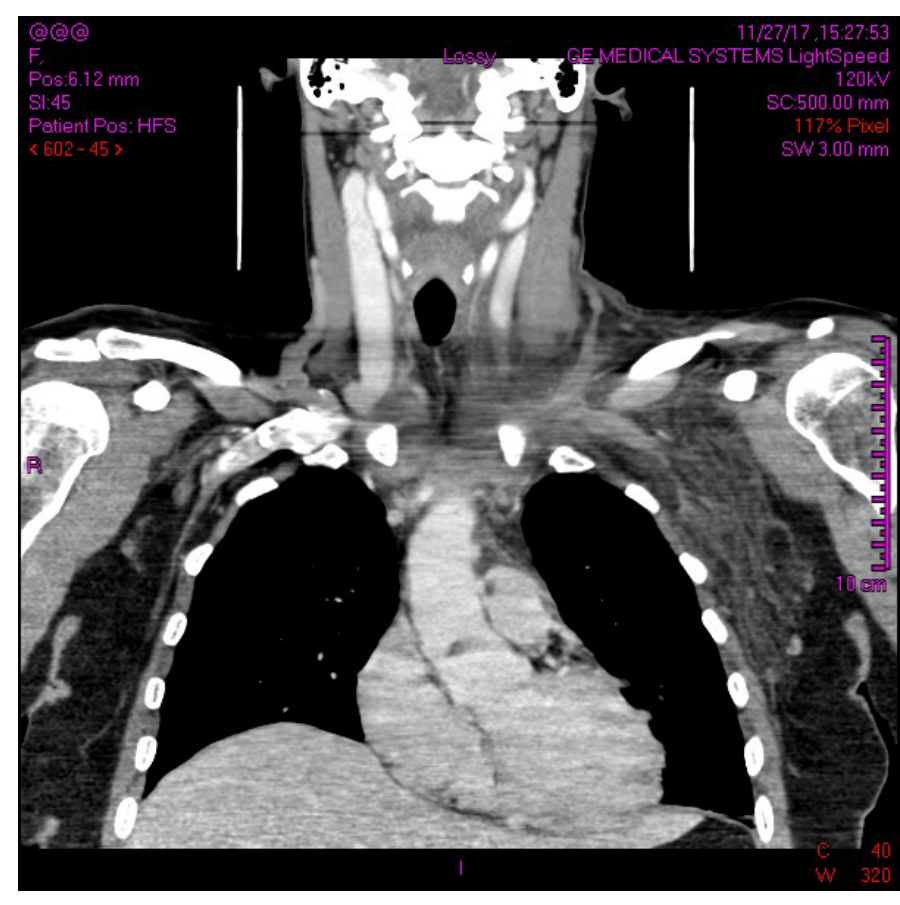

Figure 1. Initial CT scan illustrating the presence of IJV and subclavian vein thrombosis

\section{Discussion}

The Clinical presentation of IJVT is often subtle and easy to miss.

We suspected an exercise induced thrombosis due to history of prior strenuous upper limb exercise as the only risk factor identified. Previous studies have defined strenuous exercise as single unusual sport activity performed within few days prior the onset of DVT symptoms [4]. Typical activities for effort thrombosis reported in literature include weight-lifting, rowing or activities involving repetitive overhead arm movements [5].

Primary IJVT accounts for about 20\% and effort thrombosis only 3-4\% of all IJVT cases, according to a large-scale study on 1948 consecutive DVT patients [1].

${ }^{\star}$ Correspondence to: Sergei Mashkov, Surgical registrar, General Surgery, Whanganui DHB, New Zealand, E-mail: dr.mashkov@gmail.com

Received: April 19, 2019; Accepted: April 25, 2019; Published: April 29, 2019 


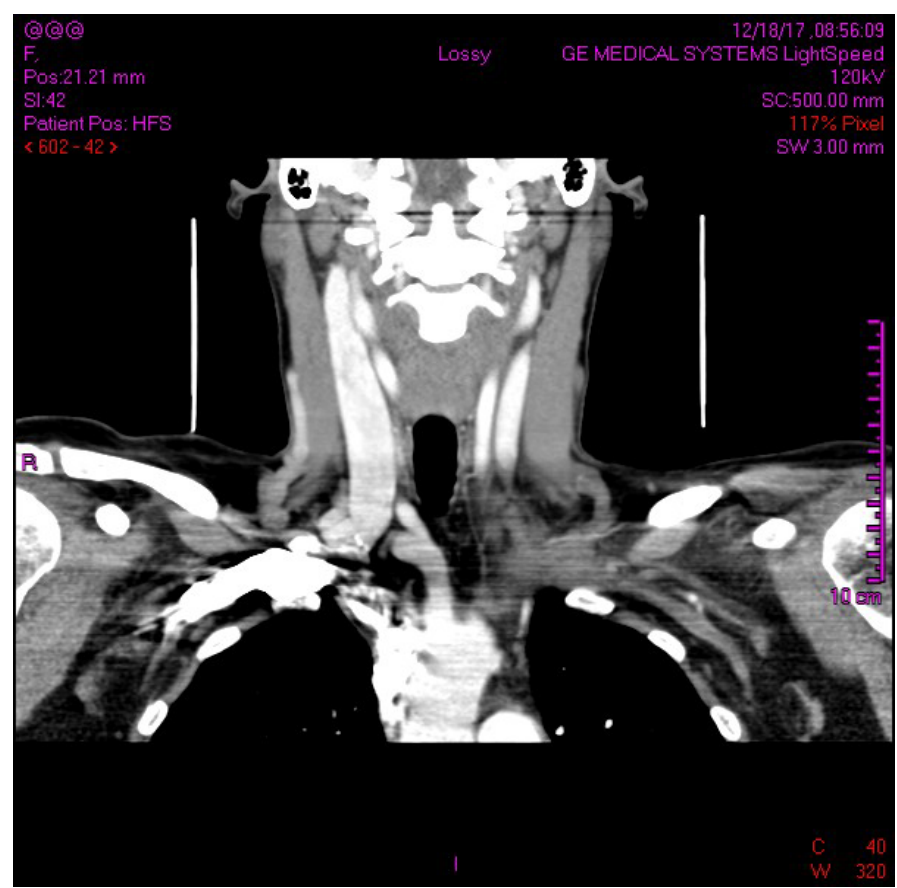

Figure 2. Subsequent CT scan illustrating the resolution of IJVT

In our case IJVT was associated with upper extremity deep vein thrombosis (UEDVT), in particular, a thrombosis of the subclavian vein.

Effort thrombosis of the IJV associated with UEDVT is often referred as Paget-Schrotter syndrome, where vigorous upper extremity activity causes microtauma to the intimal layer of the vein and leads to spontaneous thrombosis of the deep veins on the dominant arm [3].

We have followed the American College of Chest Physicians guidelines that recommends anticoagulation for a minimum of 3 months for all patients with UEDVT.

\section{Conclusion}

Primary IJVT is a rare entity with potential for serious complication. Effort thrombosis of IJV has tendency to occur in healthy female patients in their forties, who participate in professional or recreational sports. Considering there is up to $36 \%$ risk of pulmonary embolism secondary to IJVT [4], increased awareness about this condition and high risk of suspicion are the key elements for successful management of this uncommon condition.

\section{References}

1. Gbaguidi X, Janvresse A, Benichou J, Cailleux N, Levesque H, et al. (2011) Internal jugular vein thrombosis: outcome and risk factors. QJM 104: 209-219. [Crossref]

2. Corral J, Villanueva G (2016) A Case Study of Deep Vein Thrombosis of the Right Internal Jugular Vein in a Healthy 21-Year-Old Male. Case Rep Hematol p: 2016. [Crossref]

3. Boezaart AP, Haller A, Laduzenski S, Koyyalamudi VB, Ihnatsenka B, et al. (2010) Neurogenic thoracic outlet syndrome: A case report and review of the literature. Int J Shoulder Surg 4: 27-35. [Crossref]

4. Ackland H, Marshal N, Hall NDP, Walker P, Varma DK, et al. (2009) Unilateral internal jugular vein thrombosis in a young trauma patient. Injury extra 40: 246-248.

5. Illig KA, Doyle AJ (2010) A comprehensive review of Paget-Schroetter syndrome. $J$ Vasc Surg 51: 1538-1547.

Copyright: (2019 Mashkov S. This is an open-access article distributed under the terms of the Creative Commons Attribution License, which permits unrestricted use, distribution, and reproduction in any medium, provided the original author and source are credited. 